\title{
Número de Gânglios Dissecados em peças Operatórias de Pacientes Submetidos à Ressecção Cirúrgica de Câncer Colorretal. Retrospectiva de 10 anos HNSC - Tubarão - SC
}

\author{
Number of Lymph Nodes Dissecated on Operatory Samples of Patients Submited \\ to Surgical Colorectal Cancer Resection. Ten Years Retrospective Review - \\ Tubarão / SC
}

\author{
RICARDO BECKHAUSER KUHNEN ${ }^{1}$; KAISER DESOUZA KOCK ${ }^{2}$ \\ ${ }^{1}$ Acadêmico do $5^{\circ}$ ano de Medicina da Universidade do Sul de Santa Catarina (UNISUL); \\ ${ }_{2}^{2}$ Professor orientador, Professor responsável pela Disciplina de Cirurgia do Aparelho Digestivo da UNISUL, \\ Mestre em Ciências Médicas pela UFSC.
}

\begin{abstract}
KUHNEN, RB; KOCK KS. Número de Gânglios Dissecados em Peças Operatórias de Pacientes Submetidos à Ressecção Cirúrgica de Câncer Colorretal. Retrospectiva de 10 anos HNSC - Tubarão - SC. Rev bras Coloproct, 2007;27(4): 417-422.

RESUMO: No que se refere ao CCR, a análise dos linfonodos regionais é de suma importância visto que atua como fator prognóstico para a doença, determinando ou não a necessidade de terapia adjuvante, sendo que o número sugerido pela literatura varia entre 6 a 30 linfonodos identificados no espécime. Foi objetivo do trabalho determinar o número de linfonodos analisados em peças operatórios através de laudos histo-patológicos de pacientes submetidos a tratamento cirúrgico de câncer colorretal, por adenocarcinoma. A média de idade encontrada foi de 58,69 anos. Setenta e cinco pacientes eram do sexo feminino (56,8\%). A maioria se encontrava em estadio da doença T3 (75\%). 70 pacientes tiveram diagnóstico de adenocarcinoma bem diferenciado; dentre eles, 27 (42,2\%) apresentaram gânglios positivos. Conclusão: A média de gânglios dissecados foi de 11,21 / peça operatória, sendo que a probabilidade de encontrarmos nodos positivo é maior quando mais de 10 gânglios foram pesquisados. A média de linfonodos positivos foi maior quando o adenocarcinoma é do tipo indiferenciado.
\end{abstract}

Descritores: Câncer colorretal, linfonodos, análise ganglionar, metástase ganglionar.

\section{INTRODUÇÃO}

O câncer colorretal (CCR) apresenta incidência variável nos diferentes países, predominando nos economicamente mais ricos e industrializados como a América do Norte, a Europa setentrional, Nova Zelândia e Austrália. Incidências menores são registradas na América do Sul, sudoeste da Ásia, África equatorial e Índia ${ }^{1,2}$. É o quarto tumor mais freqüente no mundo, superado apenas pelos tumores de pulmão, mama e próstata ${ }^{3}$. Nos EUA é a terceira neoplasia mais comum e a $4^{\text {a }}$ causa de morte. ${ }^{4} \mathrm{~A}$ maioria dos pacientes apresenta-se com 65 anos no momento do diagnóstico ${ }^{5}$ com incidência máxima entre 60-80 anos de idade ${ }^{6}$.

No Brasil, o câncer em geral, configura-se como problema de saúde pública de dimensões nacionais. Com o aumento da expectativa de vida do povo brasileiro e com a progressiva industrialização e globalização, as neoplasias ganharam importância crescente no perfil de mortalidade do país, ocupando o segundo lugar como causa de óbito. O CCR figura entre os cinco primeiros mais freqüentes e a sua incidência não é homogênea em todo o país, com prevalência na

Trabalho realizado no Hospital N. S. da Conceição - Tubarão - Sta. Catarina.

Recebido em 17/08/2007

Aceito para publicação em 21/09/2007 
região sul e sudeste, particularmente nos Estados de São Paulo, Rio Grande do Sul e Rio de Janeiro. Ocupa o $4^{\circ}$ lugar em incidência para homens e o $3^{\circ}$ para mulheres, excluídos os tumores de pele, não-melanomas. Em relação à idade, mais de $50 \%$ dos casos manifestam-se em indivíduos com mais de 60 anos (média de 67 anos) ${ }^{1,2}$. Para o ano de 2006 a estimativa de maior incidência encontra-se no Rio Grande do Sul com 27,68 casos para cada 100.00 homens e 28,07 casos para cada 100.000 mulheres. No estado de Santa Catarina a estimativa é de 15,09 casos para cada 100.00 homens e de 16, 51 casos para cada 100.00 mulheres ${ }^{7}$.

Preconiza-se que todos os casos de neoplasias devam ser estadiados de acordo com o sistema TNM ${ }^{8}$. Esta classificação é recomendada pelos principais órgãos, tais como American Joint Committee on Cancer (AJCC), International Union Against Cancer (UICC), College of American Pathologists, Royal College of Pathologists, Commission on Cancer of the American College of Surgeons e Instituto Nacional de Câncer. Possui três principais vantagens em relação a outros métodos de estadiamento: 1. os dados são dirigidos ao processo localizado, 2. definições e regras bem claras para facilitar a utilização e 3. uso multi-disciplinar. As informações patológicas derivam da ressecção da peça cirúrgica, determinando o seguimento pós-operatório da necessidade ou não de terapia adjuvante ${ }^{9}$.

Define-se metástase ganglionar como a identificação histológica de células neoplásicas com correspondente reação. O método para identificação de linfonodos sugerido é o LNRS (lymph node revealing solution) ${ }^{10}$. A maioria das metástases ganglionares são evidenciadas em linfonodos de pequeno tamanho (inferior a $5 \mathrm{~mm}$ de diâmetro) ao passo que linfonodos maiores tendem a apresentar componentes inflamatórios $4,10,11,12$. O número de linfonodos recuperados na ressecção varia amplamente, e depende de fatores tais como: idade, variações anatômicas, da presença de linfonodos na peça, da técnica cirúrgica e o hábito do patologista em coletar todos os linfonodos existentes ${ }^{9}$, ${ }^{13}$. Falhas no exame trans-operatório ou durante análise patológica podem subestimar o número correto de linfonodos acometidos, podendo comprometer no estadiamento e prognóstico do paciente ${ }^{8}$.

É de comum acordo entre a maioria dos autores pesquisados que comprometimento ou não das cadeias ganglionares é o fator mais importante no que se refere ao estabelecimento de um prognóstico para o paciente acometido pelo CCR 10, 14, 15, 16, 17, 18, 19, 20, 21, sendo que atualmente existe uma série de sugestões em relação ao número mínimo de linfonodos que necessitem serem examinados, entretanto, parece claro que além do aspecto quantitativo, a análise qualitativa é de grande relevância para o correto prognóstico do $\mathrm{CCR}^{22}$.

Jestin e cols. ${ }^{17}$ em trabalho analisando 3375 pacientes com diagnóstico de CCR, evidenciaram que somente $64 \%$ dos casos $(n=2390)$ o número de linfonodos examinados foi mencionado pelo serviço de patologia. Nestes a mediana de gânglios analisados foi 8 (média 9,4). E somente $19 \%$ dos casos analisados seguiram a recomendação de análise mínima de 12 gânglios.

A avaliação de 12 linfonodos vem sendo aceita pelo Comitê Americano de União Contra o Câncer e pelo Congresso Mundial de Gastroenterologia como o número mínimo para o paciente ser estratificado como livre de doença metastática. Número também proposto por Bruch ${ }^{10}$, Baxter ${ }^{12}$ e Yoshimatsu ${ }^{14}$

Entretanto em um trabalho realizado junto ao serviço de Cirurgia Abdomino-Pélvica do Hospital do Câncer I - INCA foi sugerido um número mínimo de 30 linfonodos a serem analisados de modo a determinar com maior precisão a presença de metástases ganglionares. ${ }^{23}$

Tekkis e cols ${ }^{20}$ analisaram 5164 pacientes submetidos a tratamento cirúrgico de câncer colorretal em 79 hospitais na Grã-Bretanha. Foi obtida uma média de 11,7 linfonodos examinados em cada espécime. Existiu uma variabilidade, entre os 79 centros pesquisados, de 5,5 a 21,3 gânglios pesquisados. Todavia os autores afirmam que não se pode fixar um número mínimo de linfonodos a serem pesquisados.

Com base no exposto, percebemos uma variabilidade em relação à padronização do número de gânglios a serem dissecados, apesar de já estabelecida à importância da sua detecção como fator decisivo no estadiamento pós-operatório. Para tanto nos dispomos a conhecer a realidade local, visto ser este um serviço de referência na região no tratamento cirúrgico do CCR.

\section{OBJETIVO}

Determinar o número de gânglios dissecados em peças operatórias de pacientes submetidos a tratamento cirúrgico de CCR e sua associação com o grau de diferenciação celular. 
Rev bras Coloproct Outubro/Dezembro, 2007
Número de Gânglios Dissecados em Peças Operatórias de Pacientes Submetidos à Ressecção Cirúrgica de Câncer Colorretal. Retrospectiva de 10 anos HNSC - Tubarão - SC Ricardo Beckhauser Kuhnen e Cols.
Vol. 27 $\mathbf{N}^{\circ} 4$

\section{MÉTODO}

Foram analisados em estudo transversal descritivo 217 pacientes entre janeiro de 1996 até dezembro de 2006. Destes, foram selecionados 132 pacientes $(60,8 \%)$, que seguiram os critérios abaixo:

- Submetidos a tratamento cirúrgico de câncer colorretal no período compreendido entre 1997 a 2006, no município de Tubarão / SC.

- Todos os pacientes submetidos à cirurgia eletiva ou não com diagnóstico prévio de adenocarcinoma foram incluídos no estudo.

- Excluíram-se os pacientes com doença inflamatória e outros tipos de neoplasia que não adenocarcinoma e pacientes submetidos à terapia neoadjuvante

Todos os pacientes foram operados pelo pesquisador principal e o tratamento cirúrgico instituído obedeceu ao padrão convencional de ressecções, seguindo os princípios oncológicos que incluem a linfadenectomia e, no caso dos tumores retais, a ressecção do mesorreto intacto. Não foram realizadas ressecções por via laparoscópica na presente casuística.

- As peças pós-operatórias foram enviadas para laboratório de patologia, onde foram processadas e analisadas de acordo com o Manual de Padronização de Laudos Histopatológicos*.

- Todos os dados foram catalogados em forma de banco de dados e inserido no formato de planilha eletrônica no Microsoft Excel e transferidos para análise estatístico no EpiInfo.

\section{RESULTADOS}

Um total de 132 pacientes foram submetidos a tratamento cirúrgico para ressecção de CCR entre 1997 e 2006. A média de idade encontrada foi de 58,69 anos. Setenta e cinco pacientes eram do sexo feminino $(56,8 \%)$ (tabela 1). A maioria dos pacientes encontrava-se em estadio da doença T3 (75\%) (tabela 2). Setenta pacientes tiveram diagnóstico de adenocarcinoma bem diferenciado (tabela 2). Dentre eles, 27 (42,2\%) apresentaram gânglios positivos (tabela 2). Gânglios positivos foram encontrados em $44,1 \%$ e $83,3 \%$ dos casos em pacientes com diagnóstico de adenocarcinoma moderadamente diferenciado e adenocarcinoma indiferenciado respectivamente $(p-$ ANOVA $=$ 0,00011 ) (tabela 2). A média de linfonodos coletados foi de 11,21 .

Tabela 1 - Predileção por sexo.

\begin{tabular}{lll}
\hline Sexo & $\mathbf{N}$ & \% \\
\hline Homem & 57 & 43,2 \\
Mulher & 75 & 56,8 \\
\hline
\end{tabular}

$p<0,001$

Tabela 2 - Estadiamento e classificação histopatológica.

\begin{tabular}{lcccc}
\hline & $\mathbf{n}^{\mathbf{0}}$ pacientes & $\begin{array}{c}\text { média de } \\
\text { gânglios }+\end{array}$ & $\begin{array}{c}\text { pacientes } \\
\text { com nodos }+\end{array}$ & $\%$ \\
\hline Estadiamento & & & & \\
\multicolumn{1}{c}{ T1 } & 9 & 0 & 0 & 0,0 \\
T2 & 13 & 0,75 & 3 & 23,0 \\
T3 & 99 & 2,68 & 45 & 45,4 \\
T4 & 11 & $*, 81$ & 6 & 54,4 \\
Diagnóstico (Adenocarcinoma) & & 2,65 & 27 & 38,6 \\
bem diferenciado & 70 & 1,52 & 6 & 28,6 \\
bem-moderadamente diferenciado & 21 & 1,97 & 15 & 44,1 \\
moderadamente diferenciado & 34 & 11,16 & 5 & 83,3 \\
indiferenciado & 6 & 3 & 1 & 100,0 \\
adeno mucinoso & 1 & &
\end{tabular}

$* p($ ANOVA $)=0,00011$

** $p$ (qui-quadrado) $<0,00001$ 
Rev bras Coloproct Outubro/Dezembro, 2007
Número de Gânglios Dissecados em Pecas Operatórias de Pacientes Submetidos à Ressecção Cirúrgica de Câncer Colorretal. Retrospectiva de 10 anos HNSC - Tubarão - SC Ricardo Beckhauser Kuhnen e Cols.

Fazio e cols. ${ }^{24}$ reportaram 768 casos de CCR

Vinte e cinco por cento dos pacientes com diagnóstico de T2 tiveram gânglios positivos. A proporção sobe para 45,4\% e 54,4\%, respectivamente, para estadio T3 e T4 $(p=0,11)$ (tabela 3$)$.

Pacientes foram agrupados em cinco categorias de ressecção. Não houve estatística significante nas porcentagens de pacientes com gânglios positivos em relação ao tipo de procedimento cirúrgico realizado $(p-$ Kruskall-Wallis $=0,32)($ tabela 4$)$.

\section{DISCUSSÃO}

O câncer colorretal possui incidência variável nos diferentes países, com predomínio nos mais ricos e industrializados. É o quarto tumor mais freqüente no mundo e a maioria dos pacientes apresenta-se com 65 anos no momento do diagnóstico. No Brasil, figura entre os mais prevalentes com incidência preferencial nas regiões sul e sudeste. É importante que os pacientes sejam estadiados pelo sistema TNM e a avaliação ganglionar seja realizada de forma pormenorizada, pois foi consenso na literatura que comprometimento ou não das cadeias ganglionares é o fator mais importante no que se refere ao estabelecimento do prognóstico. a partir do Registro Familiar de Câncer Colo Retal de Ontário, sendo que praticamente não houve predileção por sexo ( $\mathrm{H}-49,3 \%$ e $\mathrm{M}-50,7 \%)$, a idade de diagnóstico foi superior aos 60 anos em $60,8 \%$ dos casos. Saad-Hossne e cols. ${ }^{25}$ em análise retrospectiva de pacientes portadores de CCR e demonstraram a preferência pelo sexo masculino (60\%). Justin e cols ${ }^{21}$ pesquisaram 151 pacientes submetidos à cirurgia para exérese de CCR, sendo que a maioria $(51,6 \%)$ foi composta por homens em estágio T3 $(55,6 \%)$, e $43,7 \%$ tinham gânglios positivos, com uma média de 12,8 linfonodos pesquisados. Wong e cols..$^{13} \mathrm{em}$ uma amostra de 2149 pacientes com diagnóstico de CCR demonstraram que $56 \%$ dos casos ocorreram em homens com idade entre 23 e 98 anos (média 68,7 anos) e a maioria dos pacientes tinha estadio T3 $(62 \%, \mathrm{n}=1333)$. Mahmut e cols. ${ }^{4}$ relataram o estudo sobre 179 casos de pacientes com CCR. A maioria dos pacientes foi do sexo masculino (50,8\%), com idade média de 57 anos. De acordo com sistema TNM, 44,7\% dos pacientes apresentava estadio II da doença.

Ao contrário da literatura apresentada nossa pesquisa encontrou um predomínio do sexo feminino

Tabela 3 - Proporção de linfonodos positivos de acordo com o número de gânglios analisados.

\begin{tabular}{ccccc}
\hline $\begin{array}{c}\mathbf{N}^{\mathbf{0}} \text { de nodos } \\
\text { examinados }\end{array}$ & $\mathbf{N}^{\mathbf{0}}$ de pacientes & $\begin{array}{c}\text { Pacientes } \\
\text { com nodos }+\end{array}$ & $\begin{array}{c}\% \\
\text { nodos }+\end{array}$ & Média de \\
\hline $0-4$ & 20 & 7 & 35,0 & 0,75 \\
$5-9$ & 34 & 16 & 47,1 & 1,47 \\
$10-14$ & 40 & 17 & 42,5 & 2,10 \\
$15-19$ & 18 & 10 & 55,5 & 5,50 \\
$20-24$ & 5 & 0 & 0,0 & 0,0 \\
$25-29$ & 1 & 1 & 100,0 & 21,0 \\
$\geq 30$ & 6 & 3 & 50,0 & 11,7 \\
\hline
\end{tabular}

Tabela 4 - Proporção do número de gânglios dissecados de acordo com o tipo de cirurgia.

\begin{tabular}{lcccc}
\hline Tipo de ressecção & $\mathbf{N}^{\mathbf{0}}$ de pacientes & $\begin{array}{c}\text { Pacientes } \\
\text { com nodo }+\end{array}$ & $\%$ & $\begin{array}{c}\text { média de } \\
\text { gânglios }+*\end{array}$ \\
\hline Colectomia (D / E) & 20 & 8 & 40,0 & 7,75 \\
Sigmoidectomia e RSA & 97 & 35 & 36,1 & 1,55 \\
Amputação do reto & 13 & 5 & 38,4 & 2,15 \\
Transversectomia & 2 & 1 & 50,0 & 4,00 \\
\hline
\end{tabular}

$* p($ Kruskall-Wallis $)=0,32$. 
Rev bras Coloproct Outubro/Dezembro, 2007
Número de Gânglios Dissecados em Peças Operatórias de Pacientes Submetidos à Ressecção Cirúrgica de Câncer Colorretal. Retrospectiva de 10 anos HNSC - Tubarão - SC Ricardo Beckhauser Kuhnen e Cols.
(56,8\%) com média de idade de 58,69 anos, com a maioria dos pacientes no estadio T3 (75\%), com uma média de 11,21 linfonodos pesquisados.

$\mathrm{O}$ estudo realizado por Hida e cols ${ }^{16}$ investigou 164 pacientes com CCR sendo analisados um total de 12.496 linfonodos (média de 76,2 / paciente). A proporção de metástases (número de pacientes com nodo positivo dividido pelo $\mathrm{n}^{\circ}$ total de pacientes) foi de $58,6 \%$ (96 pacientes) e a incidência de linfonodos metástaticos ( $\mathrm{n}^{\mathrm{o}}$ de gânglios + dividido pelo $\mathrm{n}^{\mathrm{o}}$ total de linfonodos) foi de 5,8\% (724 de 12.496). Em nossa pesquisa encontramos 40,9\% (54 pacientes de 132) como proporção de metástase e uma incidência de 24,38\% (334 gânglios positivos de 1390) de linfonodos metastáticos.

A pesquisa realizada por Wong e cols. ${ }^{15} \mathrm{com}-$ preendeu um total de 196 pacientes submetidos a tratamento cirúrgico de CCR e o número de linfonodos recuperados a partir de cada espécime variou de 0 a 78, com uma média de 17 gânglios. Neste estudo, 76 pacientes $(38,6 \%)$ tinham pelo menos 1 nodo positivo. Sugerindo que quanto maior o número de gânglios analisados, maior foi à chance de encontrar doença metastática. Colaborando com literatura, concluímos que quanto maior o número de gânglios pesquisados maior será a chance de encontrarmos nodos positivos, pois quando analisamos entre 0-4 linfonodos a média de linfonodos positivos foi de 0,75 , entre $10-14$ foi de 2,10, entre $15-19$ foi 5,50 e mais de 30 foi de 11,7 (tabela 3 ).

\section{CONCLUSÕES}

A média de gânglios dissecados foi de 11,21 / peça operatória, sendo que a probabilidade de encontrarmos nodos positivos é maior quando mais de 10 gânglios foram pesquisados.

A média de linfonodos positivos foi maior quando o adenocarcinoma é do tipo indiferenciado.

ABSTRACT: Lymph node analysis is very important in colorectal cancer since it is a prognosis factor for the disease, which will either determine the needs of adjuvant therapy, or not. The amount of identified lymph nodes per specimen suggested by the literature varies from 6 to 30. The purpose of this work was to show the amount of lymph nodes analyzed from operatory subjects by means of the histopathology records from patients undergoing colorectal cancer surgical treatment from adenocarcinoma. The average age found was 58,69 years. Seventy five patients were females $(\mathbf{5 6 , 8} \%)$. Most patients were found to be in phase T3 of the disease $(75 \%)$. Sixty four patients had a very differentiated adenocarcinoma. Among them, $27(42,2 \%)$ showed positive nodes. Conclusion: The average of dissected lymph nodes was of 11,21 / specimen. Being that the probability of finding positive node was greater when $\mathbf{1 0}$ or more nodes were analyzed. The average of positive lymph node was greater in undifferentiated adenocarcinoma.

Key words: Colorectal cancer, lymph nodes, nodes analysis, nodes metastasis.

\section{REFERÊNCIAS}

* Manual de Padronização de Laudos Histopatológicos / Sociedade Brasileira de Patologia ; [editores: Carlos Eduardo Bacchi, Marcello Fabiano de Franco, Paulo Cirilo Cardoso de Almeida] - 3ed - São Paulo : Reichmann \& Autores Editores, 2005.

1. Gama, A.H - Câncer Colorretal - A importância de sua prevenção, Arq Gastroenterol, v. 42 - no. 1 - jan. /mar. 2005.

2. Malheiros, A.P.R. - Resultado do tratamento cirúrgico do câncer colo-retal em doentes de idade até 64 anos e de 65 anos ou mais. Revista Brasileira de Coloproctologia 2005; 25 (2): 128-136.

3. Rohde, L e cols. - Câncer colorretal In : Rotinas em Cirurgia Digestiva, Ed Artmed, Porto Alegre, p.187-92, 2005.

4. Mahmut, G e cols. - What is the optimal number of lymph nodes to be dissected in colorectal cancer surgery ?, Tumori $2005 ; 91: 168-72$.
5. Moesinger, R.C. - Diagnosis and emerging therapies in the treatment of colorectal cancer. Johns Hopkins Advanced Studies in Medicine - Oncology January 2006 ; 6 (1):30-9 .

6. Cotran, R.C ; Kumar, V ; Collins, T - O Trato GastroIntestinal In: Robins: Patologia Estrutural e Funcional . Ed Guanabara-Koogan, Rio de Janeiro, 2000.

7. http://www.inca.gov.br/estimativa/2006/index.asp?link= mapa.asp\&ID=6, acessado em 15/02/2006

8. Sarli, L. e cols. - Number of limph nodes examined and prognosis of TNM stage II colorectal cancer . European Journal of Cancer 2005 ; n 41 : p 272-9 .

9. Compton, C.C ; Greene,F.L - The Staging of Colorectal Cancer: 2004 and Beyond CA, Cancer J Clin 2004 ; v 54 (6):295-308 .

10. Bruch, H.P. e cols. - Actual standards and controversies on operative technique and limph node dissection in colorectal cancer . Langenbeck's Arch Surgery 1999 ; v.384 : 167-175. 
11. Bilchik, A. - More (nodes) + More (analyses) $=$ Less (Mortality): Challenging the therapeutic equation for earlystage colon cancer. Annals of Surgical Oncology $2003 ; 10$ (3): 203-205 .

12. Baxter, N.N. e cols. - Lymph node evaluation in Cancer Colorectal Pacients: A population based study, Journal of the National Cancer Institute Feb 2005 ; 97 (3) : 219-25.

13. Wong, J.H. e cols. - Assessing the quality of colorectal cancer staging: documenting the process in improving the staging of node-negative colorectal cancer. Archives of Surgery Sep. 2005 ; 140(9) : 881-6.

14. Yoshimatsu, K e cols. - How many lymph nodes should be examined in Dukes 'B colorectal cancer? Determination the basis of cumulative survival rate. Hepatogastroenterology NovDec 2005 ; 52(66) : 1703-6.

15. Wong, J.H. e cols. - Number of nodes examined and staging accuracy in colorectal carcinoma . Journal of Clinical Oncology Sep / 1999, 17 (9) : 2896-2900 .

16. Hida, J e cols. - Number versus distribuition in classifyng regional lymph node metastases from colon cancer. J Am College of Surgeon Aug / 2005 ; 201 (2) : 217-22.

17. Jestin, $\mathrm{P}$ e cols. - Cancer staging and survival in colon cancer is dependent on the quality of the pathologists specimen examination. Eur. Journal of Cancer Sept. 2005; 41 (14) : 2071-8 .

18. Cserni, G ; Tzrján, M. Bori, R. - Distance of limph nodes from the tumor, na importante feature in colorectal câncer specimens . Arch Pathol Lab Med Feb / 2001 ; v. 125 : 246-49.
19. Mainprize, K.S. e cols. - How many lymph nodes to stage colorectal carcinoma? J. Clin. Pathol 1998 ; 51 : p.165-166.

20. Tekkis, P.P. e cols. - A national study on lymph node retrival in resection surgery for coloretal cancer. Diseases of colon \& rectum November $2006 ; 49: 1673-1683$.

21. Kim, J, e cols - Number of lymph nodes examined and its impact on colorectal cancer staging October 2006 ; 10 : 902905.

22. Cserni, G - The influence of nodal size on the staging of colorectal carcinomas J. Clin. Pathol. 2002 ; 55 : 386-390.

23. Kesley, R. e cols. - Qual o número ideal de linfonodos a serem estudados na peça cirúrgica de adenocarcinomas do reto? Revista Brasileira de Coloproctologia Setembro 2004 ; 24 (supl. 1), p 88 .

24. Fazio, L. e cols. - Association between colonic screening, subject characteristics and stage of colorectal cancer . Am J Gastroenterology 2005, 100: 2531-2539.

25. Saad-Hossne, R. e cols. - Estudo retrospectivo de pacientes portadores de câncer colorretal atendidos na Faculdade de medicina de Botucatu no período de 2000-2003. Revista Brasileira de Coloproctologia 2005 ; 25 (1):31-37.

\section{Endereço para correspondência: \\ RICARDO BECKHAUSER KUHNEN}

Av Marcolinio Martins Cabral, 2075 Ed Interclínicas - $7^{\circ}$ andar

Tubarão / SC

88075-001

Fone (Fax): (48) 3631-1500 / 3631-1560 